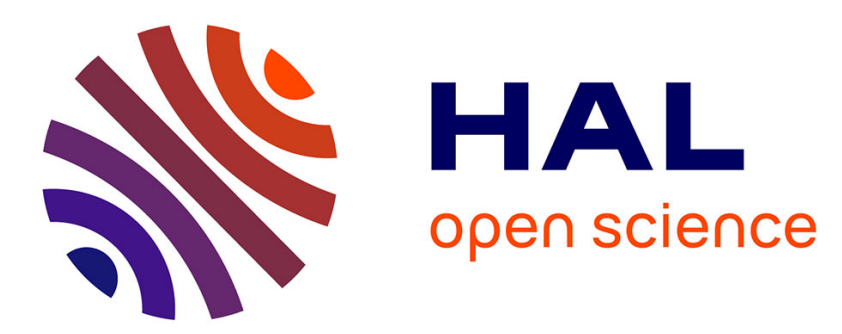

\title{
Micro-macro modelling of stress-dependent anisotropic magnetoresistance
}

\author{
A Bartók, Laurent Daniel, A Razek
}

\section{To cite this version:}

A Bartók, Laurent Daniel, A Razek. Micro-macro modelling of stress-dependent anisotropic magnetoresistance. Journal of Physics D: Applied Physics, 2011, 44 (13), pp.135001. 10.1088/0022$3727 / 44 / 13 / 135001$. hal-00606296

\section{HAL Id: hal-00606296 \\ https://hal.science/hal-00606296}

Submitted on 6 Jul 2011

HAL is a multi-disciplinary open access archive for the deposit and dissemination of scientific research documents, whether they are published or not. The documents may come from teaching and research institutions in France or abroad, or from public or private research centers.
L'archive ouverte pluridisciplinaire HAL, est destinée au dépôt et à la diffusion de documents scientifiques de niveau recherche, publiés ou non, émanant des établissements d'enseignement et de recherche français ou étrangers, des laboratoires publics ou privés. 


\title{
Micro-macro modeling of stress-dependent anisotropic magnetoresistance
}

\author{
A. Bartók, L. Daniel and A. Razek \\ E-mail: andras.bartok@lgep.supelec.fr \\ Laboratoire de Génie Electrique de Paris (LGEP) \\ CNRS (UMR 8507) - SUPELEC - UPMC Paris 6 - Univ Paris-Sud 11, \\ 11 rue Joliot-Curie, Plateau de Moulon, 91192 Gif-sur-Yvette, France
}

\begin{abstract}
.
Anisotropic magnetoresistance (AMR) is the basic phenomenon of a spread class of sensors. AMR effect has a strong mechanical stress dependence. Micromagnetic simulations are often used for modeling the magnetoresistance of ferromagnetic materials, but these approaches do not allow to investigate macroscopic effects (for example behavior of a polycrystal under stress) due to the high number of interactions and degrees of freedom. On the other hand macroscopic phenomenological approaches fail in describing the main role of microstructure on the effective behavior. In this work a micro-macro model is proposed to describe the effect of stress on the AMR in ferromagnetic polycrystals. Results are discussed and compared to experimental data from the literature.
\end{abstract}

PACS numbers: 73.43.Qt, 75.80.+q, 75.30.Gw, 75.60.-d

Submitted to: J. Phys. D: Appl. Phys. 


\section{Introduction}

The anisotropic magnetoresistance (AMR) effect in ferromagnetic materials (FM) was first discovered by William Thomson in 1857. Due to its high sensitivity and flexibility of design it is still used in a wide array of sensors for measurement of Earth's magnetic field (electronic compass) [1], for electrical current measuring (by measuring the magnetic field created around the conductor) [2], for traffic detection [3] and for linear position and angle sensing [4].

The electrical resistivity in FM depends on the angle between the direction of electrical current and orientation of the magnetization in the material. This anisotropic dependence lies in spin-orbit coupling. It is fundamentally related to the larger probability of s-d scattering for electrons traveling parallel to the magnetization [5]. As the magnetization rotates, the electron cloud about each nucleus deforms slightly and this deformation changes the amount of scattering undergone by the conduction electrons in their passage through the lattice [6]. Thus the AMR effect is strongly dependent on the local magnetization in the material. In that sense it has some similarities with the magnetostriction effect as discussed later in this paper. On the other hand, owing to the magnetic domain structure of FM, the distribution of magnetization within FM is very heterogeneous. This is the reason why numerical models for AMR effect are mostly based on micromagnetic calculations [7, 8, 9]. In these approaches the number of degrees of freedom and interactions are growing quickly with the number of magnetic moments, so that these simulations can only address small volumes corresponding to a limited number of domains. If the effect of the microstructure on the overall AMR properties is to be investigated - for example the effect of crystallographic texture in polycrystalline media - these methods are not relevant.

It is also known that the AMR effect has a strong mechanical stress dependence [10]. Indeed mechanical stress applied to a magnetic media changes the distribution of domain orientations. As a consequence it modifies the local resistivity, and thus the overall resistivity. This effect of stress on magnetoresistance is for instance used as the basis for a particular type of strain measurement gauges [11].

Some macroscopic phenomenological models for AMR effect have also been proposed $[12,13,14]$ but they cannot account for microstructure or composition related effects. They have to be identified on macroscopic measurements and cannot be used as predictive tools for material design.

An intermediate approach, standing between micromagnetic and macroscopic modeling, would be useful in order to provide a design tool sensitive to complex microstructural effects, and notably accounting for the crystallographic texture. Indeed this microstructural parameter can be controlled and significantly modifies the overall AMR properties of FM. In this work a micro-macro model for stress-dependent AMR is proposed. This model is based on a magneto-elastic multiscale model allowing the definition of the local stress and magnetic field in heterogeneous materials from the knowledge of the macroscopic loading. A phenomenological law for AMR effect is then used at the local scale. A homogenization step is finally performed to define the macroscopic change in resistivity. This model can naturally account for the effect of stress on the overall AMR effect, and includes the influence of microstructural parameters such as the crystallographic texture.

The paper is divided into three parts. In the first part the micro-macro model is detailed. In the second part the approach is validated thanks to experimental 
measurements on iron, nickel and permalloy polycrystals taken from the literature. The approach is finally applied in the third part to the investigation of the effect of crystallographic texture on AMR.

\section{Micro-macro modeling}

AMR effect depends on the local magnetization orientation, itself depending on local magnetic field and stress (at the magnetic domain scale). Owing to the heterogeneity of materials, stress and magnetic field are not uniform within the material. Their local values have to be determined through an appropriate micro-macro scheme. Once these values for the local loading are known, the magnetic domain structure has to be determined, in order to define the local magnetization (at the domain scale). A model for the AMR effect can then be applied. The overall response of the material (polycrystalline scale) is then obtained through appropriate averaging operations. Thus, the model proposed in this paper is based on a three-scale description (polycrystal, single crystal, magnetic domain) and follows the scheme presented in Figure 1.

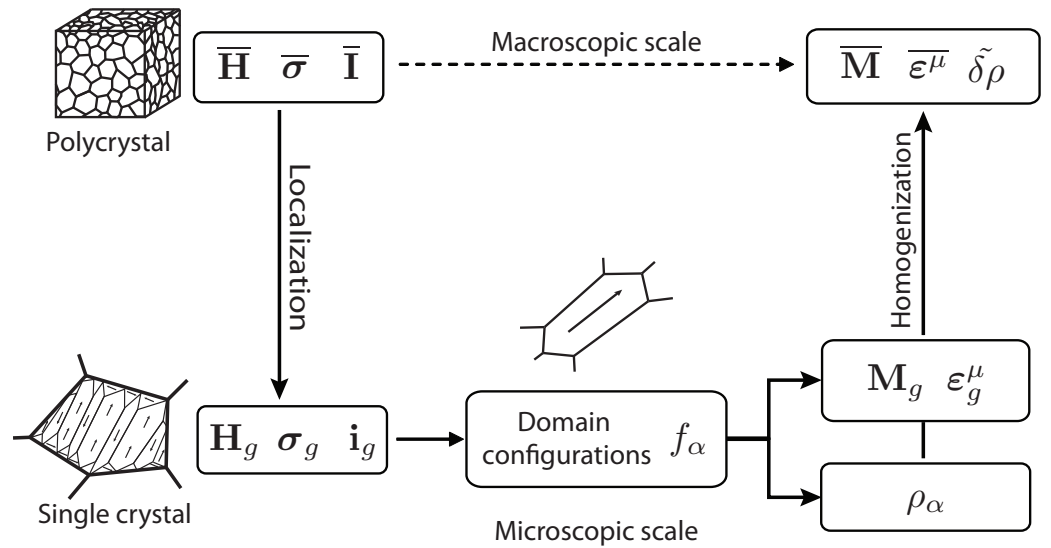

Figure 1. Modeling strategy

The uppermost calculation scale in the model - called macroscopic scale - is the polycrystalline representative volume element (RVE) which is seen as an assembly of single crystals or grains $(g)$ with respect to a given orientation function. The intermediate scale - called mesoscopic scale - is the single crystal or grain, that is seen as a collection of magnetic domains $(\alpha)$ with given magnetization orientation. The lowest scale - called microscopic scale - is the magnetic domain, that is an area with uniform magnetostriction strain $\ddagger$ and magnetization.

The main steps of this model are divided as follows.

(i) The localization steps aims at defining the local loading (magnetic field $\mathbf{H}_{\alpha}$, stress $\boldsymbol{\sigma}_{\alpha}$ and current $\mathbf{i}_{\alpha}$ ) at the microscopic scale as a function of the macroscopic loading (magnetic field $\overline{\mathbf{H}}$, stress $\overline{\boldsymbol{\sigma}}$ and current $\overline{\mathbf{I}}$ ). The loading at the mesoscopic scale (magnetic field $\mathbf{H}_{g}$, stress $\boldsymbol{\sigma}_{g}$ and current $\mathbf{i}_{g}$ ) is calculated as an intermediate step. These localization steps highly depend on the microstructure of the material.

$\ddagger$ magnetostriction strain is the spontaneous strain undertaken by magnetic materials. 
(ii) The microscopic magneto-elastic model allows to define in a statistical way the domain configuration, introducing as an internal variable the volumetric fraction $f_{\alpha}$ of domains with orientation $\boldsymbol{\alpha}$ in a grain $g$. In each domain, depending on the considered orientation $\boldsymbol{\alpha}$, the magnetization $\mathbf{M}_{\alpha}$ and magnetostriction strain $\varepsilon_{\alpha}^{\mu}$ are known.

(iii) The microscopic AMR model allows to define the local resistivity $\left(\rho_{\alpha}\right)$ depending on the magnetization orientation $\boldsymbol{\alpha}$ in the considered domain.

(iv) The homogenization step allows to retrieve the overall response of the material at the polycrystal scale (magnetization at the macroscopic scale $\overline{\mathbf{M}}$, macroscopic magnetostriction strain $\overline{\varepsilon^{\mu}}$ and the variation of the macroscopic resistivity $\tilde{\delta \rho}$ ) depending on the local values of the response at the microscopic scale.

These steps are detailed hereafter. The magneto-elastic part of the model is based on a reversible magneto-elastic model previously published $[15,16]$.

\subsection{Localization step}

The simplest assumption to define the microscopic loading $\left(\mathbf{H}_{\alpha}, \boldsymbol{\sigma}_{\alpha}, \mathbf{i}_{\alpha}\right)$ as a function of the macroscopic loading $(\overline{\mathbf{H}}, \overline{\boldsymbol{\sigma}}, \overline{\mathbf{I}})$ would be to consider uniform field hypotheses. Under such hypotheses the localization rules are very simple $\left(\mathbf{H}_{\alpha}=\overline{\mathbf{H}}, \boldsymbol{\sigma}_{\alpha}=\overline{\boldsymbol{\sigma}}\right.$, $\mathbf{i}_{\alpha}=\overline{\mathbf{I}}$ ). However due to the heterogeneity of the materials, these assumptions are often inappropriate.

1.1.1. Macro-meso scale transition In a polycrystal the susceptibility from one grain to another can vary very significantly. For instance in pure iron the permeability of a grain can vary up to $70 \%$ at $400 \mathrm{~A} / \mathrm{m}$ or or $60 \%$ at $2000 \mathrm{~A} / \mathrm{m}$ depending on its relative orientation with respect to the magnetic field [17]. This heterogeneity results in a significant heterogeneity of the magnetic field within the material. In the case of polycrystals, the self-consistent scheme is known to provide satisfying results. The macro-meso localization rule is written as follows [16]:

$$
\mathbf{H}_{g}=\overline{\mathbf{H}}+\frac{1}{3+2 \chi^{m}}\left(\overline{\mathbf{M}}-\mathbf{M}_{g}\right)
$$

$\overline{\mathbf{M}}$ and $\mathbf{M}_{g}$ are the magnetization respectively at the macroscopic and mesoscopic scales. In the case of self-consistent hypothesis $\chi^{m}$ is the overall magnetic susceptibility of the material.

The elastic response to a given mechanical loading also significantly differs from one grain to another in a polycrystal. As an example in the case of pure iron the Young's modulus can vary up to $115 \%$ depending on the crystallographic orientation (see the elastic constants in Table 1). The self-consistent localization scheme is known to provide satisfying estimates for polycrystalline media [18]. Under such hypotheses, the macro-meso localization rule can be written in the following form [16]:

$$
\boldsymbol{\sigma}_{g}=\mathcal{B}_{\sigma}: \overline{\boldsymbol{\sigma}}+\mathcal{L}^{\mathrm{inc}}:\left(\overline{\varepsilon^{\mu}}-\varepsilon_{g}^{\mu}\right)
$$

$\overline{\varepsilon^{\mu}}$ and $\varepsilon_{g}^{\mu}$ are the magnetostriction strain respectively at the macroscopic and mesoscopic scale. $\mathcal{B}_{\sigma}$ denotes the so-called concentration tensor and $\mathcal{L}^{\text {inc }}$ is a tensor accounting for elastic incompatibilities due to magnetostriction. The way to calculate these fourth order tensor can be found in $[18,16]$ and is briefly re-called in Appendix A. 
In the case of the electrical resistivity, and as will be shown in the following, the heterogeneity is weak. Depending on the orientation of the single crystal, the electrical resistivity does not vary more than a few percent. This is why we applied uniform electric current conditions.

$$
\mathbf{i}_{g}=\overline{\mathbf{I}}
$$

1.1.2. Meso-micro scale transition In the case of the localization rules from the grain to the domain scale, an accurate definition of the microstructure, namely the magnetic domain structure, would be requisite $\S$. This information is unknown. For the sake of simplicity we assumed uniform magnetic field, uniform strain and uniform current within the single crystal. However in the proposed microscopic magneto-elastic model, the mean values at the single crystal scale are often sufficient data.

\subsection{Microscopic magneto-elastic model}

The magneto-elastic model for the single crystal is derived from [15]. The single crystal is seen as an assembly of magnetic domains. The potential energy of a domain $\alpha$ is written:

$$
W_{\alpha}=W_{\alpha}^{K}+W_{\alpha}^{\sigma}+W_{\alpha}^{H}
$$

where $W_{\alpha}^{K}$ denotes the magneto-crystalline energy, $W_{\alpha}^{\sigma}$ denotes the elastic energy and $W_{\alpha}^{H}$ denotes the magneto-static energy.

In the case of cubic crystallographic structure the magneto-crystalline energy can be written

$$
W_{\alpha}^{K}=K_{1}\left(\alpha_{1}^{2} \alpha_{2}^{2}+\alpha_{2}^{2} \alpha_{3}^{2}+\alpha_{3}^{2} \alpha_{1}^{2}\right)+K_{2}\left(\alpha_{1}^{2} \alpha_{2}^{2} \alpha_{3}^{2}\right)
$$

where $K_{1}$ and $K_{2}$ denote the magneto-crystalline anisotropy constants of the cubic crystal and $\boldsymbol{\alpha}={ }^{t}\left[\begin{array}{lll}\alpha_{1} & \alpha_{2} & \alpha_{3}\end{array}\right]$ the direction cosines of the magnetization $\left(\mathbf{M}_{\alpha}=M_{s} \boldsymbol{\alpha}\right.$ with $M_{s}$ the saturation magnetization of the material).

Under uniform strain hypotheses the elastic energy can be written [16]:

$$
W_{\alpha}^{\sigma}=-\sigma_{g}: \varepsilon_{\alpha}^{\mu}
$$

In the case of cubic crystallographic symmetry, the magnetostriction strain tensor $\varepsilon_{\alpha}^{\mu}$ can be written as

$$
\varepsilon_{\alpha}^{\mu}=\frac{3}{2}\left(\begin{array}{ccc}
\lambda_{100}\left(\alpha_{1}^{2}-\frac{1}{3}\right) & \lambda_{111} \alpha_{1} \alpha_{2} & \lambda_{111} \alpha_{1} \alpha_{3} \\
\lambda_{111} \alpha_{1} \alpha_{2} & \lambda_{100}\left(\alpha_{2}^{2}-\frac{1}{3}\right) & \lambda_{111} \alpha_{2} \alpha_{3} \\
\lambda_{111} \alpha_{1} \alpha_{3} & \lambda_{111} \alpha_{2} \alpha_{3} & \lambda_{100}\left(\alpha_{3}^{2}-\frac{1}{3}\right)
\end{array}\right)
$$

where $\lambda_{100}$ and $\lambda_{111}$ are the magnetostrictive constants of the single crystal.

The magneto-static energy of a domain is written as

$$
W_{\alpha}^{H}=-\mu_{0} \mathbf{M}_{\alpha} \cdot \mathbf{H}_{\alpha}
$$

where $\mu_{0}$ is the vacuum permeability.

$\S$ except for the electric current since the hypotheses of weak heterogeneity of the resistivity is still valid. 
We then introduce the volumetric fractions $f_{\alpha}$ of domains with magnetization orientation $\boldsymbol{\alpha}[19,20,21,16]$. These internal variables are obtained through the numerical integration of the following Boltzmann-type relation [15]:

$$
f_{\alpha}=\frac{\exp \left(-A_{s} \cdot W_{\alpha}\right)}{\int_{\alpha} \exp \left(-A_{s} \cdot W_{\alpha}\right) d \alpha}
$$

where $A_{s}$ is an adjustable parameter that can be deduced from low field measurement of the anhysteretic magnetization curve [16] $\left(A_{s}=\frac{3 \chi_{0}}{\mu_{0} M_{s}^{2}}\right.$ where $\chi_{0}$ is the initial anhysteretic susceptibility of the material).

The magnetostriction strain and the magnetization over the single crystal are defined by an averaging operation over the single crystal (volume $V_{g}$ ):

$$
\begin{aligned}
& \varepsilon_{g}^{\mu}=\left\langle\varepsilon^{\mu}\right\rangle_{g}=\frac{1}{V_{g}} \int_{\alpha} \varepsilon^{\mu} d V=\sum_{\alpha} f_{\alpha} \varepsilon_{\alpha}^{\mu} \\
& \mathbf{M}_{g}=\langle\mathbf{M}\rangle_{g}=\frac{1}{V_{g}} \int_{\alpha} \mathbf{M} d V=\sum_{\alpha} f_{\alpha} \vec{M}_{\alpha} .
\end{aligned}
$$

If needed, the elastic strain $\varepsilon_{g}^{e}$ in the single crystal can be easily calculated from Hooke's law (Equation (12)) using the single crystal stiffness tensor $\mathcal{C}_{g}$. This elastic strain is superimposed to the magnetostriction strain to obtain the total strain of the single crystal $\left(\varepsilon=\varepsilon^{e}+\varepsilon^{\mu}\right)$.

$$
\varepsilon_{g}^{e}=\mathcal{C}_{g}^{-1}: \sigma_{g}
$$

At this stage, the macroscopic magnetization and magnetostriction strain could also be calculated thanks to an averaging operation over the whole volume of the RVE.

\subsection{Single-domain model of $A M R$}

Let $\boldsymbol{\beta}={ }^{t}\left[\beta_{1} \beta_{2} \beta_{3}\right]$ be the direction cosines determining the orientation of the current used for measuring the electrical resistance $\left(\boldsymbol{\alpha}={ }^{t}\left[\begin{array}{lll}\alpha_{1} & \alpha_{2} & \alpha_{3}\end{array}\right]\right.$ are still the direction cosines of the magnetization in the considered domain). The general expression for the magnetoresistance in any direction of a cubic crystal can be written in a series form of $\boldsymbol{\alpha}$ and $\boldsymbol{\beta}$ [13]. Döring used the following form [10] for cubic crystals with negative magnetocrystalline anisotropy constant $\| K_{1}$ (such as nickel and $\mathrm{Fe}_{11} \mathrm{Ni}_{89}$ permalloy):

$$
\begin{aligned}
\rho_{\alpha} & =\rho_{0}\left[1+k_{1}\left(\alpha_{1}^{2} \beta_{1}^{2}+\alpha_{2}^{2} \beta_{2}^{2}+\alpha_{3}^{2} \beta_{3}^{2}-\frac{1}{3}\right)\right. \\
& +2 k_{2}\left(\alpha_{1} \alpha_{2} \beta_{1} \beta_{2}+\alpha_{2} \alpha_{3} \beta_{2} \beta_{3}+\alpha_{3} \alpha_{1} \beta_{3} \beta_{1}\right) \\
& +k_{3}\left(s-\frac{1}{3}\right) \\
& +k_{4}\left(\alpha_{1}^{4} \beta_{1}^{2}+\alpha_{2}^{4} \beta_{2}^{2}+\alpha_{3}^{4} \beta_{3}^{2}+\frac{2 s}{3}-\frac{1}{3}\right) \\
& \left.+2 k_{5}\left(\alpha_{1} \alpha_{2} \beta_{1} \beta_{2} \alpha_{3}^{2}+\alpha_{2} \alpha_{3} \beta_{2} \beta_{3} \alpha_{1}^{2}+\alpha_{3} \alpha_{1} \beta_{3} \beta_{1} \alpha_{2}^{2}\right)\right]
\end{aligned}
$$

in wich $s=\alpha_{1}^{2} \alpha_{2}^{2}+\alpha_{2}^{2} \alpha_{3}^{2}+\alpha_{3}^{2} \alpha_{1}^{2}, \rho_{0}$ is the resistivity in the demagnetized state and $k_{1}, k_{2}, k_{3}, k_{4}, k_{5}$ are material constants. For a crystal with positive magnetocrystalline anisotropy constant $K_{1}$ (such as iron) the expression is the same except that the term $k_{3} / 3$ is absent.

$\|$ in that case easy magnetization directions are $\langle 111\rangle$ directions.

व in that case easy magnetization directions are $\langle 100\rangle$ directions. 


\subsection{Effective properties}

The macroscopic magnetization and strain are obtained through an averaging operation over the whole volume $V$ of the RVE.

$$
\begin{aligned}
& \overline{\mathbf{M}}=\langle\mathbf{M}\rangle_{V}=\left\langle\mathbf{M}_{g}\right\rangle_{V} \\
& \bar{\varepsilon}=\langle\varepsilon\rangle_{V}=\left\langle\varepsilon^{e}+\varepsilon^{\mu}\right\rangle_{V}=\left\langle\varepsilon_{g}\right\rangle_{V}
\end{aligned}
$$

If needed, the macroscopic magnetostriction strain can be obtained using the following relation $[16]$ :

$$
\overline{\varepsilon^{\mu}}=\left\langle{ }^{t} \mathcal{B}_{\sigma}: \varepsilon^{\mu}\right\rangle_{V}=\left\langle{ }^{t} \mathcal{B}_{\sigma}: \varepsilon_{g}^{\mu}\right\rangle_{V}
$$

Since the local electric conductivity $\varsigma_{\alpha}$ in a domain is known $\left(\varsigma_{\alpha}=1 / \rho_{\alpha}\right)$, the effective macroscopic conductivity $\tilde{\varsigma}$ can be obtained through a self-consistent approach, applying the classical Bruggeman relation [22, 23, 17]. $\tilde{\varsigma}$ is solution of Equation (17) that can be solved easily using a fixed point method.

$$
\tilde{\varsigma}=\frac{\left\langle\frac{\varsigma_{\alpha}}{2 \tilde{\varsigma}+\varsigma_{\alpha}}\right\rangle_{V}}{\left\langle\frac{1}{2 \tilde{\varsigma}+\varsigma_{\alpha}}\right\rangle_{V}}
$$

where the operation $\langle.\rangle_{V}$ is an averaging operation over the whole volume of the RVE. The effective resistivity $\tilde{\rho}$ is deduced from the effective conductivity $(\tilde{\rho}=1 / \tilde{\varsigma})$. In the following the variation of the macroscopic resistivity $\tilde{\delta \rho}$ (Equation (18)) will be plotted. It can be noticed from Equations (13) and (17) that $\tilde{\delta \rho}$ does not depend on the value of $\rho_{0}$.

$$
\tilde{\delta \rho}=\frac{\tilde{\rho}-\rho_{0}}{\rho_{0}}
$$

\section{Validation on isotropic polycrystals}

The proposed micro-macro approach has been validated on isotropic iron, nickel and permalloy $\left(\mathrm{Fe}_{11} \mathrm{Ni}_{89}\right)$ polycrystals.

\subsection{Model parameters}

The mechanical and magnetic characteristics of iron, nickel and a permalloy $\left(\mathrm{Fe}_{11} \mathrm{Ni}_{89}\right)$ single crystal were used $^{+}$for the calculations. At the single crystal scale, for the calculation of the volumetric fractions $f_{\alpha}$, a 10242 orientation $(\alpha)$ data file was used [15]. The material constants are defined in Table 1 and Table 2. The distribution function for crystal orientation in the case of isotropic polycrystals has been obtained by a regular zoning of the crystallographic space [16]. The corresponding pole figures are given in Figure 2.

+ The accurate data of the constants of Döring expression of $\mathrm{Fe}_{11} \mathrm{Ni}_{89}$ was not found. The constants of the $\mathrm{Fe}_{15} \mathrm{Ni}_{85}$ permalloy were used instead. 


\begin{tabular}{|l|l|l|l|l|l|}
\hline Coefficient & $M_{S}$ & $K_{1}, K_{2}$ & $\lambda_{100}, \lambda_{111}$ & $A_{s}$ & $C_{11}, C_{12}, C_{44}$ \\
\hline Unit & $A / m$ & $k J / m^{3}$ & - & $m^{3} / J$ & $G P a$ \\
\hline Iron $[10,6,24]$ & $1.71 \times 10^{6}$ & $42.7,15$ & $21,-21\left(\times 10^{-6}\right)$ & 0.0020 & $238,142,232$ \\
\hline Nickel $[10,25]$ & $4.91 \times 10^{5}$ & $-5.7,-2.3$ & $-45.9,-24.3\left(\mathrm{x} 10^{-6}\right)$ & 0.016 & $250,160,118$ \\
\hline $\mathrm{Fe}_{11} \mathrm{Ni}_{89}[10,26]$ & $7.50 \times 10^{5}$ & $-1,-2$ & $-15,-10\left(\times 10^{-6}\right)$ & 0.032 & $243,148,122$ \\
\hline
\end{tabular}

Table 1. Physical constants used for the modeling

\begin{tabular}{|l|l|l|l|l|l|}
\hline Coefficient & $k_{1}$ & $k_{2}$ & $k_{3}$ & $k_{4}$ & $k_{5}$ \\
\hline Iron $[10]$ & 0.00153 & 0.00593 & 0.00194 & 0.00053 & 0.00269 \\
\hline Nickel $[10]$ & 0.0654 & 0.0266 & -0.032 & -0.054 & 0.020 \\
\hline $\mathrm{Fe}_{15} \mathrm{Ni}_{85}[27]$ & 0.0518 & 0.0478 & -0.0243 & -0.0139 & 0.0259 \\
\hline
\end{tabular}

Table 2. Constants of Döring expression
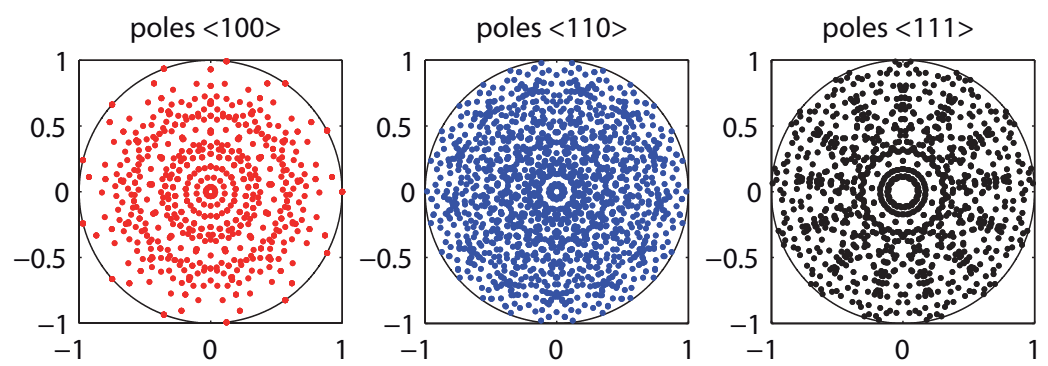

Figure 2. Pole figures for an isotropic polycrystal obtained from a regular zoning of the crystallographic space

\subsection{Prediction of the AMR effect}

The similarity in the phenomena of magnetostriction and magnetoresistance has been known for a long time. This similarity is linked to the strong dependence of both phenomena on the local magnetization state and thus on the magnetic domain configuration. Our model is based on a micro-macro approach of the magnetoelastic behavior of the materials which can give the magnetostriction $(\lambda(H))$ and the magnetoresistive curves $(\delta \rho(H))$. From these results the relation between the change of resistivity and the magnetostriction strain can be easily obtained. It is illustrated in the case of isotropic nickel and pure iron polycrystals without external stress and compared with experimental data from the literature [10] in Figure 3 and 4. These figures plot the effective magnetostriction strain as a function of the effective change in resistivity for parallel configuration (magnetic field and electrical current are parallel). Experimental observations show that magnetostriction strain first increases with magnetoresistance and then decreases in the case of iron, and magnetostriction decreases continuously in the case of nickel. The different behavior of these materials results from the different sign in their material constants (magnetostriction and Döring expression). The experimental curve is accurately predicted by the model in the case of nickel. The experimental curve for iron is qualitatively predicted, but quantitatively overestimated. It is shown by the dot plot in Figure 4 that a macroscopic tension of amplitude $25 \mathrm{MPa}$ applied in the direction of the magnetic field provides a numerical result closer to the experimental observation. This point shows that a residual stress 
in the material could explain the discrepancies in the case of the iron specimen.

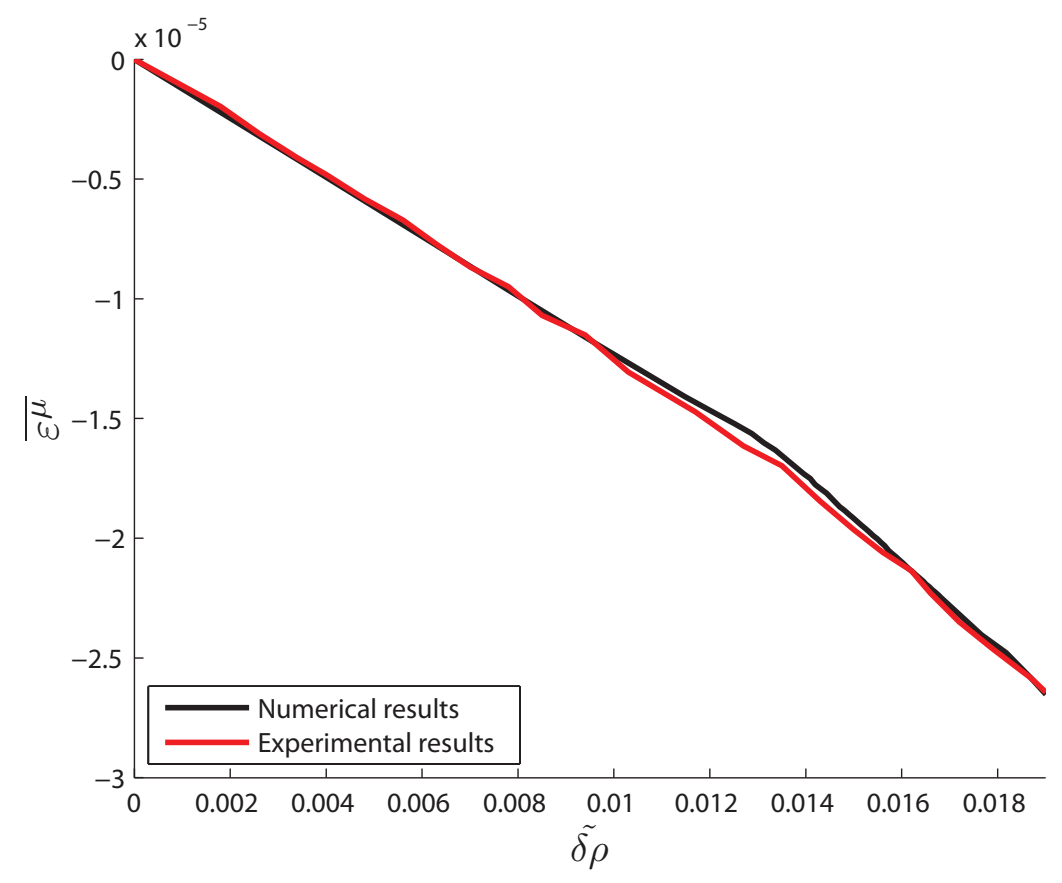

Figure 3. Magnetostriction strain of nickel polycrystal as a function of the change of resistivity (current and applied magnetic field are parallel) - experimental data [10] and obtained numerical results

\subsection{Prediction of the effect of stress on the $A M R$}

In order to study the effect of an applied uniaxial stress on the magnetoresistance, modeling results were compared to experimental results [10] on a permalloy $\left(\mathrm{Fe}_{11} \mathrm{Ni}_{89}\right)$ polycrystal. In this case the $\tilde{\delta} \rho(B)$ curves were calculated from the combination of $B(H)$ and $\tilde{\delta} \rho(H)$ modeling results. This permalloy has negative magnetostriction so that the tension orients its domains perpendicularly to the direction of tension and this effect decreases the initial resistivity. The comparison between numerical and experimental results (Figure 5) gives very satisfying results. It shows the nonlinear stress-dependence as well. The stress decreases the initial resistivity and increases the slope of the curves which is important in the sensor application (higher sensibility in low field measurement). The model can predict the effect of a multiaxial stress as well but these results are not presented in this paper due to the lack of experimental results for validation.

\section{Investigation of crystallographic texture effect}

The magnetoresistance in a single crystal is strongly anisotropic. Figure 6 shows the change of resistivity in a pure iron single crystal as a function of the angle between its easy magnetization direction $\langle 100\rangle$ and the applied magnetic field in the $\{011\}$ crystallographic plane. The change in resistivity (under no applied stress) can 


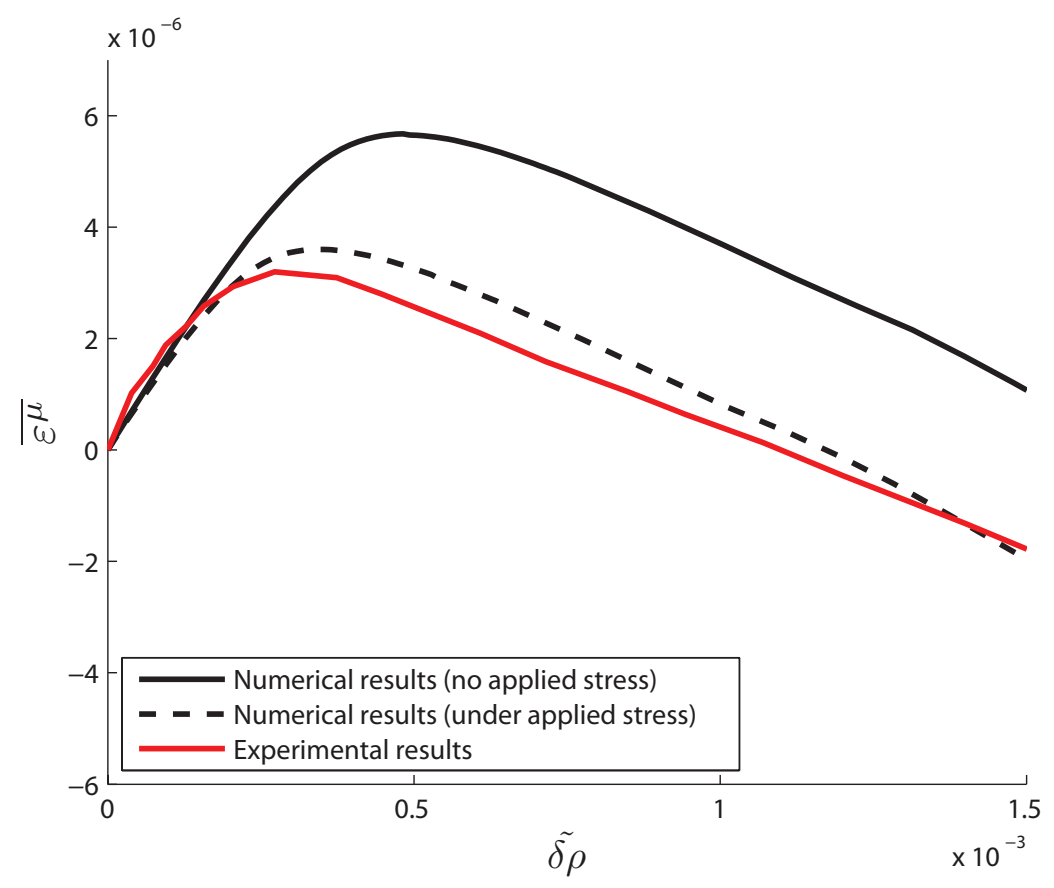

Figure 4. Magnetostriction strain of iron polycrystal as a function of the change of resistivity (current and applied magnetic field are parallel) - experimental data [10] and obtained numerical results (dashed line: under $25 \mathrm{MPa}$ tension applied in the direction of magnetic field)
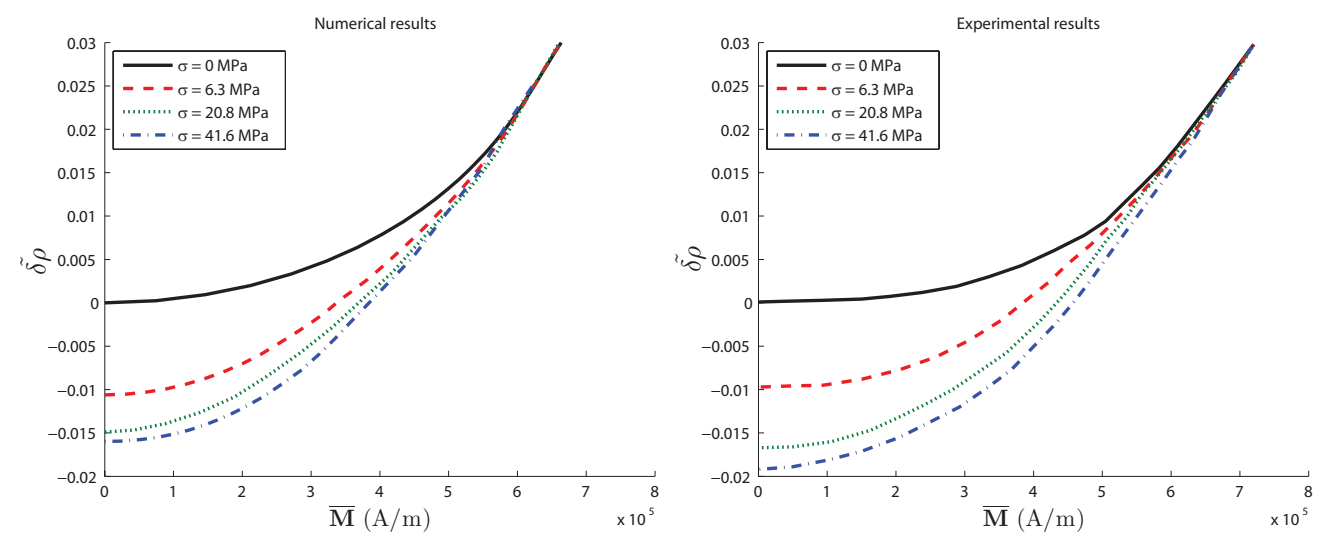

Figure 5. Change of resistivity (current and applied magnetic field are parallel) with change of magnetization of Permalloy polycrystals $\left(\mathrm{Fe}_{11} \mathrm{Ni}_{89}\right)$, effect of the level of applied uniaxial stress (compression) - obtained numerical results (left) and experimental data [10] (right)

vary up to several hundred percents depending on the orientation of the magnetic field with respect to the crystal orientation, both in parallel and perpendicular configurations. As a consequence, the AMR effect can be expected to be very sensitive to crystallographic texture. 


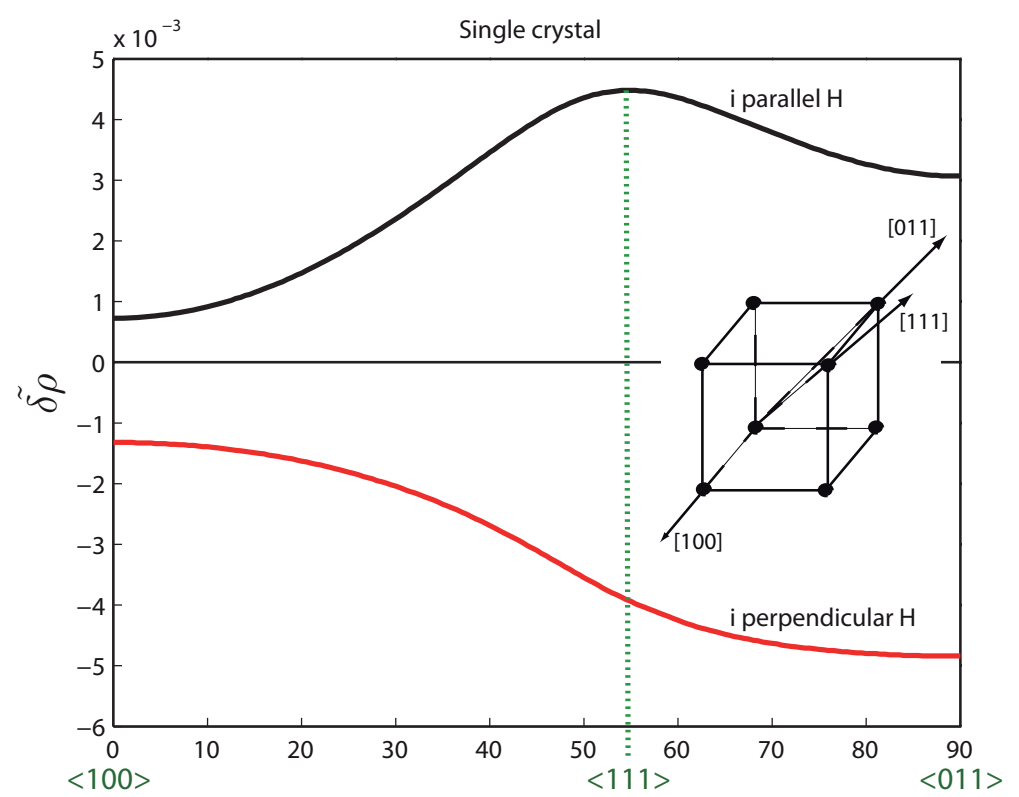

Angle between the $<100>$ direction and the applied magnetic field (in degrees)

Figure 6. Change of resistivity as a function of the angle between the $\langle 100\rangle$ direction and the applied magnetic field $\left(10^{5} \mathrm{~A} / \mathrm{m}\right)$ in the $\{011\}$ crystallographic plane - pure iron single crystal in parallel (parallel magnetic field and electrical current) and perpendicular (perpendicular magnetic field and electrical current) configurations

In order to investigate this effect, the crystallographic texture of an Armco specimen (pure iron), known as isotropic, has been obtained from electron backscattered diffraction (EBSD) measurement. The corresponding pole figures are given in Figure 7. It shows a weak texture compared with the calculated isotropic crystal orientation distribution used previously and showed in Figure 2.
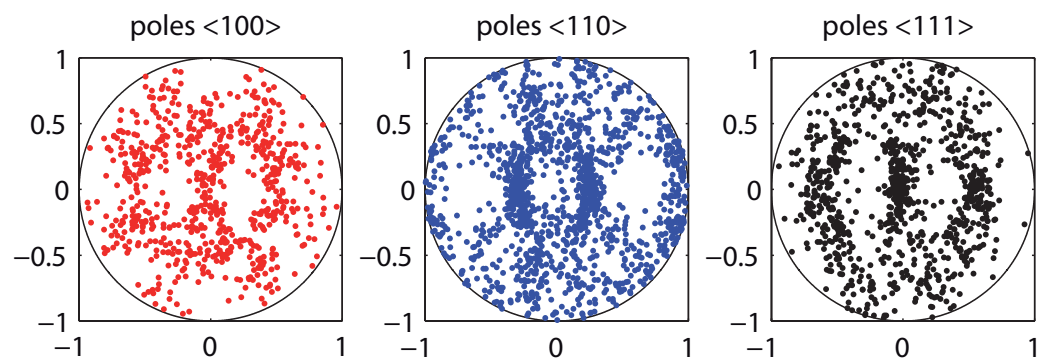

Figure 7. Pole figures of an Armco steel obtained from EBSD measurement

The prediction of the AMR effect for pure iron using this latter crystallographic texture has been compared to the prediction using the isotropic orientation data file. Figure 8 shows the change of resistivity in the parallel current/applied magnetic field configuration as a function of current orientation in the polycrystal. In the case of Armco specimen two planes (XY and YZ) have been investigated. 
It is shown that even for this very weakly textured material the magnetoresistance can vary up to $10 \%$ depending on the orientation of the sollicitation (parallel configuration). The magnetoresistance is confirmed to be strongly dependent on crystallographic texture.

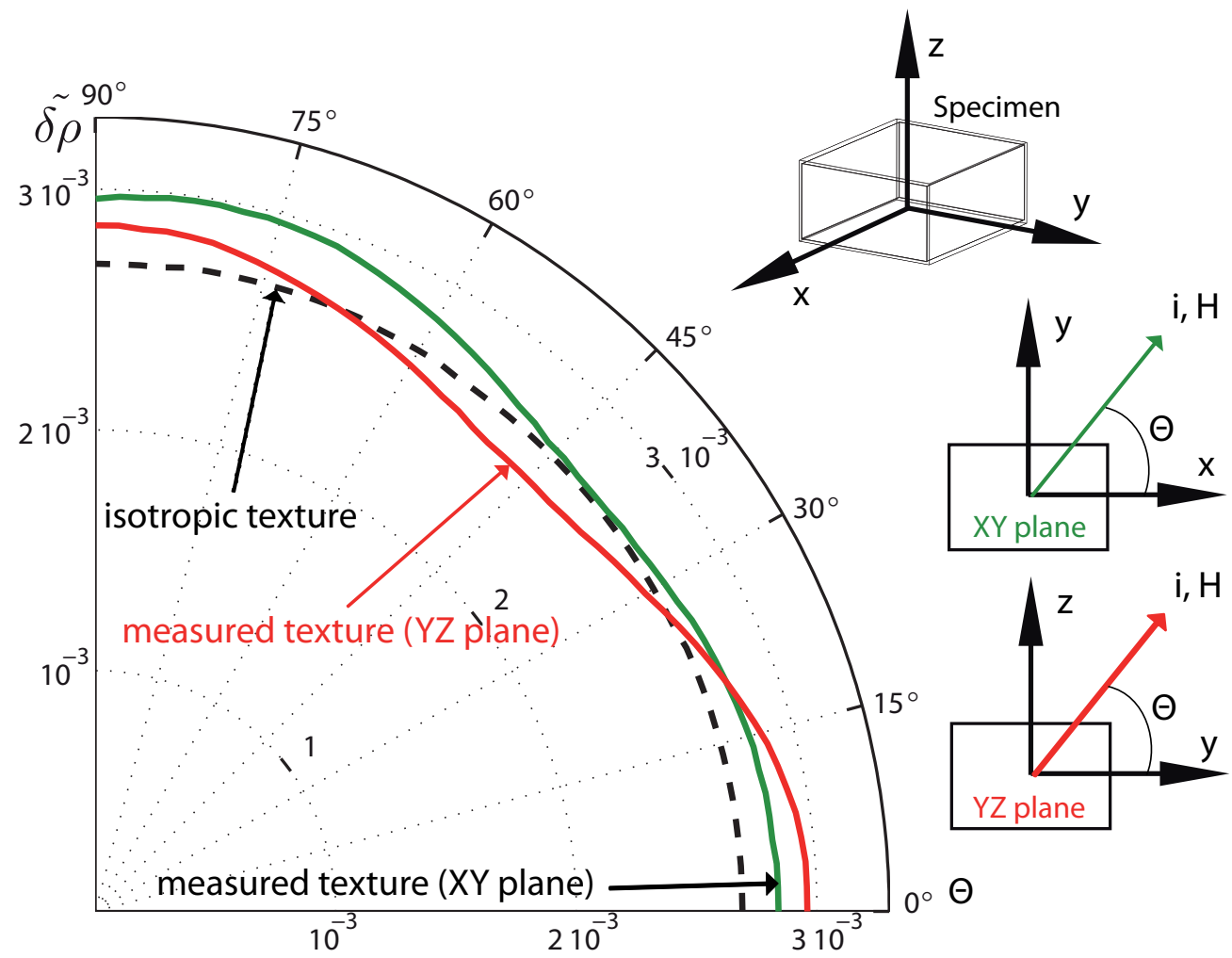

Figure 8. Change of resistivity (current and applied magnetic field are parallel) with change of the orientation of the applied magnetic field $\left(10^{5} \mathrm{~A} / \mathrm{m}\right)$ for pure iron polycrystal using an isotropic texture (dotted line) and texture data from EBSD measurement in different planes (lines)

\section{Conclusion}

A micro-macro model for the effect of stress on the anisotropic magnetoresistance has been presented. It is based on a description of the magneto-mechanical coupling at several scales (domain, single crystal, polycrystal). The behavior of iron, nickel and a permalloy $\left(\mathrm{Fe}_{11} \mathrm{Ni}_{89}\right)$ polycrystal has been calculated. Numerical results have been compared with experimental results from the literature with very satisfying agreement. This model enables to investigate the effect of crystallographic texture on AMR effect and it highlights the strong influence of crystallographic texture on it. It also enables to investigate the effect of stress, and notably multiaxial stress on magnetoresistance. The development of micro-macro models accounting for microstructure and stress dependence of effective magnetoresistive properties should allow the design of high precision AMR devices. 


\section{Appendix A: Calculation of mechanical localization operators}

We briefly give hereafter the way to obtain the fourth order tensors $\mathcal{B}_{\sigma}$ and $\mathcal{L}^{\text {inc }}$ appearing in section 1.1. More detailed explanations can be found in $[18,16]$.

$\mathcal{B}_{\sigma}$ is defined by Equation (19) that introduces the single crystal stiffness tensor $\mathcal{C}_{g}$, the polycrystal effective stiffness tensor $\tilde{\mathcal{C}}$ and the strain localization tensor $\mathcal{A}_{\sigma}$.

$$
\mathcal{B}_{\sigma}=\mathcal{C}_{g}: \mathcal{A}_{\sigma}: \tilde{\mathcal{C}}^{-1}
$$

$\mathcal{A}_{\sigma}$ is defined by Equation (20) where $\mathcal{C}^{*}$ is the so-called Hill constraint tensor [18] that can be obtained from the Eshelby tensor $\mathcal{S}^{E}$ according to Equation (21), $\mathcal{I}$ being the fourth order identity tensor.

$$
\begin{aligned}
& \mathcal{A}_{\sigma}=\left(\mathcal{C}_{g}+\mathcal{C}^{*}\right)^{-1}:\left(\tilde{\mathcal{C}}+\mathcal{C}^{*}\right) \\
& \mathcal{C}^{*}=\tilde{\mathcal{C}}:\left(\mathcal{S}^{E^{-1}}-\mathcal{I}\right)
\end{aligned}
$$

$\mathcal{L}^{\text {inc }}$ is defined by Equation $(22)$.

$$
\mathcal{L}^{\text {inc }}=\mathcal{C}_{g}:\left(\mathcal{C}_{g}+\mathcal{C}^{*}\right)^{-1}: \mathcal{C}^{*}
$$

\section{Acknowledgments}

The authors are greatly indebted to Dr. Anne-Laure Helbert (ICMMO, Univ ParisSud) for the EBSD measurement of the Amrco steel.

\section{References}

[1] J. Vcelak, P. Ripka, J. Kubik, A. Platil, and P. Kaspar. AMR navigation systems and methods of their calibration. Sensors and Actuators A, 123-124:122, 2005.

[2] P. Mlejnek, M. Vopalensky, and P. Ripka. AMR current measurement device. Sensors and Actuators A, 141:649, 2008.

[3] Application Note. Vehicle detection and compass applications using AMR magnetic sensors. Technical Report AN218, Honeywell SSEC, www.ssec.honeywell.com, 2005.

[4] D. J. Adelerhof and W. Geven. New position detectors based on AMR sensors. Sensors and Actuators, 85:48, 2000.

[5] J. Smit. Magnetoresistance of ferromagnetic metals and alloys at low temperatures. Physica, 17:612, 1951.

[6] B. D. Cullity. Introduction to Magnetic Materials. Addison-Wesley Publishing Company, London, 1972.

[7] A. O. Adeyeyea and R. L. White. Magnetoresistance behavior of single castellated $\mathrm{Ni}_{80} \mathrm{Fe}_{20}$ nanowires. J. Appl. Phys., 95:2025, 2004.

[8] M. Hafner and et al. Theory of anisotropic magnetoresistance in atomic-sized ferromagnetic metal contacts. Phys. Rev. B, 79:140410(R), 2009.

[9] S. Serrano-Guisan, K. Rott, G. Reiss, and H. W. Schumacher. Inductive and magneto-resistive measurements of gilbert damping in $\mathrm{Ni}_{81} \mathrm{Fe}_{19}$ thin films and microstructures. J. Phys. D: Appl. Phys., page 164015, 2008.

[10] R. M. Bozorth. Ferromagnetism. Van Nostrand, Princeton, NJ., 1951.

[11] M. Sonehara, M. Shinohara, T. Sato, T. Yamasawa, and K. Miura. Strain sensor using stress-magnetoresistance effect of NiFe/MnIr exchange-coupled magnetic film. J. Appl. Phys., 107:718, 2010.

[12] W. Kwiatkowski, M. Stabrowski, and S. Tumariski. Numerical analysis of the shape anisotropy and anisotropy dispersion in thin film permalloy magnetoresistors. IEEE Trans. Magn., MAG-19:2502, 1983.

[13] T.R. McGuire and R.I. Potter. Anisotropic magnetoresistance in ferromagnetic 3d alloys. IEEE Trans. Magn., 11:1018, 1975. 
[14] J. Li, S. L. Li, Z. W. Wu, S. Li, H. F. Chu, J. Wang, Y. Zhang, H. Y. Tian, and D. N. Zheng. A phenomenological approach to the anisotropic magnetoresistance and planar hall effect in tetragonal $\mathrm{La}_{2} / 3 \mathrm{Ca}_{1 / 3} \mathrm{Mno}_{3}$ thin films. J. Phys.: Condens. Matter, page 146006, 2010.

[15] L. Daniel and N. Galopin. A constitutive law for magnetostrictive materials and its application to Terfenol-d single and polycrystals. Eur. Phys. J. Appl. Phys., 42:153, 2008.

[16] L. Daniel, O. Hubert, N. Buiron, and R. Billardon. Reversible magneto-elastic behavior: A multiscale approach. J. Mech. Phys. Solids, 56:1018, 2008.

[17] L. Daniel and R. Corcolle. A note on the effective magnetic permeability of polycrystals. IEEE Trans. Magn., 43:3153, 2007.

[18] R. Hill. Continuum micro-mechanics of elastoplastic polycrystals. J. Mech. Phys. Solids, 13:213, 1965.

[19] L. Néel. Les lois de l'aimantation et de la subdivision en domaines élémentaires d'un monocristal de fer. J. Phys. Radiat., 5:241, 1944.

[20] S. Chikazumi. Introduction to ferromagnetic materials. Addison-Wesley, 1997.

[21] N. Buiron, L. Hirsinger, and R. Billardon. A multiscale model for magneto-elastic couplings. J. Phys. IV, 9:139-141, 1999.

[22] D.A.G. Bruggeman. Berechnung verschiedener physikalischer konstanten von heterogenen substanzen. Annalen der Physik, 5:636, 1935.

[23] D. Stroud. Generalized effective-medium approach to the conductivity of an inhomogeneous material. Phys. Rev. B, 12:3368, 1975.

[24] F. A. McClintock and A. S. Argon. Mechanical Behavior of Materials. Addison-Wesley Publishing Company, New-York, 1966.

[25] G. Hausch and H. Warlimont. Single crystalline elastic constants of ferromagnetic face centered cubic Fe-Ni invar alloys. Acta Metal., 21:401, 1973.

[26] A. Kanrar and U. S. Ghosh. The variation of elastic constants of nickel-iron single crystal alloys from 78.76 to 300 K. J. Phys. Chem. Solids, 44:457-462, 1983.

[27] L. Berger and S. A. Friedberg. Magnetoresistance of a permalloy single crystal and effect of 3d orbital degeneracies. Phys. Rev., 165:670, 1968. 